\title{
Graded microstructures of Al-Li-Mg-Zn-Cu entropic alloys under supergravity
}

\author{
Ruixuan $\mathrm{Li}^{1,2}$, Zhe Wang ${ }^{3}$, Zhancheng Guo ${ }^{3}$, Peter K. Liaw ${ }^{4}$, Tao Zhang ${ }^{5}$, Lugee $\mathrm{Li}^{5}$ and \\ Yong Zhang ${ }^{1,2^{*}}$
}

\begin{abstract}
Investigating the microstructures and properties of gradient materials has been regarded as a promising way to accelerate the identification of optimal compositions for applications. Herein, a supergravity method is applied to prepare the graded entropic alloys $\mathrm{Al}-\mathrm{Zn}-\mathrm{Li}-\mathrm{Mg}$ - $\mathrm{Cu}$. Through carefully optimizing the experimental conditions, the graded microstructures and hardness values appear after the supergravity technique. The morphology of the alloy significantly changes from the bulk intermetallics to eutectic structures along the supergravity force direction, which results from the crushed and graded aluminum oxide combined with the extremelystrong force. The results show that with this supergravity method, a performance-enhanced alloy can potentially be achieved through the centrifugation in a short time span and thus it paves the way for designing and synthesizing entropic alloys with intriguing properties.
\end{abstract}

Keywords: entropic alloys, supergravity, graded microstructures, grain refinement, viscosity

\section{INTRODUCTION}

Graded materials can be commonly seen in biological structures, such as bamboo, turtle shell, and bone. Analogously, the concept of graded materials is now widely used by the materials science for a series of materials exhibiting a spatial gradient in microstructures or compositions [1,2]. Carburizing and nitriding are firstly used in ancient China to improve the comprehensive performance of steels [3], which is considered as the prototype of graded materials. In the past few decades, such kinds of materials, which have a gradual transition from the softer and tougher core to the hardened edge, are proved to have better properties than the homogeneous materials [4-7], such as higher damage tolerance, higher plasticity, and higher strength.

Powder metallurgy [8,9], self-propagating high-temperature synthesis (SHS) [10], vapor deposition [11], and many other methods have been successfully employed to prepare graded materials. Furthermore, the supergravity technique has been proved to be useful for the fabrication of graded materials based on the density difference [1214], which has a simple mechanism and simple experimental operations, compared with the aforementioned techniques. This method has been successfully used in the metallurgy of the impurity separation and elements concentration [15-19], but its application in high-entropy alloys is rarely reported.

In our previous research, the microstructure of $\mathrm{Al}_{x} \mathrm{CoCrFeNi}$ (in molar ratio, $x=0,0.3,0.5,0.75$, and 1 ) high-entropy alloys obtained by the combustion synthesis under high gravity was investigated, and a graded composition was predicted [20]. Nowadays, light-weight multicomponent alloys have attracted much attention [21-24]. Yang et al. [25] reported that the phase formation rules of low-density quinary alloys from the Al-Li$\mathrm{Mg}-(\mathrm{Zn}, \mathrm{Cu}, \mathrm{Sn})$ system was different from that of the traditional multicomponent alloys with transition elements, and the configurational entropy was not sufficient enough to stabilize the disordered solid solution phases. As a result, the light-weight multicomponent alloys containing $\mathrm{Al}, \mathrm{Li}, \mathrm{Mg}$ exhibit an extremely complex multiphase structure, which leads to a poor mechanical

\footnotetext{
${ }^{1}$ State Key Laboratory for Advanced Metals and Materials, University of Science and Technology Beijing, Beijing 100083, China

${ }^{2}$ Beijing Key Laboratory for Magneto-Photoelectrical Composite and Interface Science, University of Science and Technology Beijing, Beijing 100083, China

${ }^{3}$ State Key Laboratory of Advanced Metallurgy, University of Science and Technology Beijing, Beijing 100083, China

${ }^{4}$ Department of Materials Science and Engineering, The University of Tennessee, Knoxville, TN, 37996, USA

${ }^{5}$ Dongguan EON Institute for Advanced Materials, Dongguan 523662, China

*Corresponding author (email: drzhangy@ustb.edu.cn)
} 


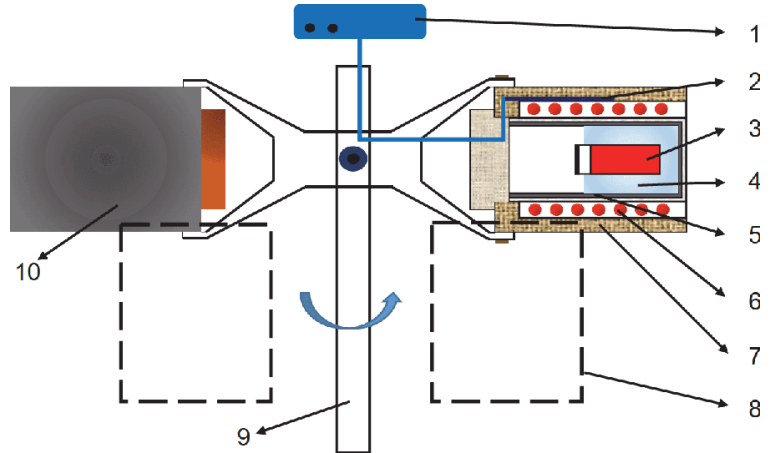
(1) Temperature controller
(6) Resistance wire
(2) Thermocouple
(7) Insulating layer
(3) Molten metal
(8) Heating furnace in resting position
(4) Fixity
(5) Crucible
(9) Centrifugal axis
(10) Counterweight

Figure 1 Schematic of the centrifugal apparatus.

property, and hence graded materials are a great choice to improve the comprehensive properties of them.

In this paper, we propose a simple method to fabricate light-weight graded materials via supergravity. Compared with the conventional method just as powder metallurgy, this high-throughput method can greatly shorten the processing period and simply reduce the energy consumption through centrifugation. This paper mainly discusses the graded microstructures of $\mathrm{AlZn}_{0.4} \mathrm{Li}_{0.2} \mathrm{Mg}_{0.2^{-}}$ $\mathrm{Cu}_{0.2}$ entropic alloys prepared by the supergravity technique, their properties as well as the corresponding mechanisms.

\section{EXPERIMENTAL SECTION}

A supergravity field is created by a centrifugal force, which is produced through the rotation by a centrifugal apparatus shown in Fig. 1. When the apparatus is rotating around the axis, the samples in the furnace is in the supergravity field and subjected to the supergravity force (also called the centrifugal force), which points from the closer end to the centrifugal axis to the far end. The centrifugal apparatus consists of a temperature controller, a furnace, a counterweight, and some other parts. To generate a stable and adjustable supergravity field, the heating furnace and the counterweight are controlled in the same weight and fixed symmetrically onto a centrifugal rotor, and then they rotate from vertical resting state to the horizontal working state when the centrifugal rotor starts running. In this experiment, the supergravity direction is horizontal. Moreover, the heating furnace is heated by a resistance wire. The isothermal zone is $80 \mathrm{~mm}$ long, and the temperature is controlled by a program controller. The supergravity coefficient $(G)$ is calculated as the ratio of the super-gravitational acceleration to normal gravitational acceleration via Equation (1):

$G=\frac{\sqrt{g^{2}+\left(\omega^{2} r\right)^{2}}}{g}=\frac{\sqrt{g^{2}+\left(n^{2} \pi^{2} r / 900\right)^{2}}}{g}$,

where $\omega$ is the angular velocity $\left(\mathrm{rad} \mathrm{s}^{-1}\right) ; n$ is the rotating speed of the centrifuge $\left(\mathrm{r} \mathrm{s}^{-1}\right) ; r$ is the distance from the centrifugal axis to the sample (m); and $g$ is normal gravitational acceleration. $G=500$, and $r=0.25-0.28 \mathrm{~m}$ in this experiment.

The raw materials used in the experiments were the pure aluminum (99.7\% purity) ingot, pure zinc (99.9\% purity) ingot, magnesium lithium alloy (Mg-20wt\%Li), and pure copper (99.9\% purity) ingot. The Al-Zn-Li-Mg$\mathrm{Cu}$ alloy ingots with a nominal chemical composition of $\mathrm{AlZn}_{0.4} \mathrm{Li}_{0.2} \mathrm{Mg}_{0.2} \mathrm{Cu}_{0.2}$ (in at.\%) were melted in a vacuum induction melting (VIM) furnace under an argon atmosphere, and then they were put into the furnace of the centrifugal apparatus. Under the resistance heating and high gravity of $500 \mathrm{~g}$, the alloy ingots remelted above the melting point, after which they were divided into three groups and centrifuged for different times. Details are listed in the Table 1. Finally, the light-weight entropic alloys with the graded microstructure were obtained, and then machined and polished for later experiments.

Cutting along the central axis, five representative positions of each samples were selected, and their microstructures, compositions, as well as properties were characterized by a scanning electron microscope (SEM) with the energy-dispersive X-ray spectroscope (SEMEDX), X-ray diffraction (XRD), X-ray fluorescence (XRF), and the micro-hardness tester. The sample surface

Table 1 Experimental details of the three samples

\begin{tabular}{ccccc}
\hline & Holding temperature $\left({ }^{\circ} \mathrm{C}\right)$ & $\begin{array}{c}\text { Centrifugation time during } \\
\text { insulation }(\mathrm{min})\end{array}$ & $\begin{array}{c}\text { Cooling rate } \\
\left({ }^{\circ} \mathrm{C} \text { min }{ }^{-1}\right)\end{array}$ & $\begin{array}{c}\text { Centrifugation time during } \\
\text { cooling }(\mathrm{min})\end{array}$ \\
\hline Sample 1 & 680 & 0 & 7 & 0 \\
Sample 2 & 680 & 5 & 7 & 0 \\
Sample 3 & 680 & 5 & 7 & 5 \\
\hline
\end{tabular}


was mechanically polished before being examined. It should be mentioned here that the quantitative measurement of the Li concentration profile is difficult because the $\mathrm{Li}$ atomic ratio is only $1 \mathrm{wt} . \%$, which is approaching the detection limitation of EDX and XRF. Moreover, some Li may be lost during the preparation, which further increases the difficulty in detection. Fortunately, Li has not shown an obvious role in the alloy. Thus, it is not stressed in the following discussion.

\section{RESULTS}

\section{Characterization of $\mathrm{AlZn}_{0.4} \mathrm{Li}_{0.2} \mathrm{Mg}_{0.2} \mathrm{Cu}_{0.2}$ light-weight entropic alloys}

Fig. $2 \mathrm{a}$ and $\mathrm{b}$ shows the photographs of $\mathrm{AlZn}_{0.4} \mathrm{Li}_{0.2} \mathrm{Mg}_{0.2^{-}}$ $\mathrm{Cu}_{0.2}$ (Sample 1) before and after centrifugation, respectively. Some defects, such as shrinkage holes and porosities, can be seen in Fig. 2b, which probably results from the low fluidity and the nature of casting during cooling. There is also some aluminum oxide on the surface of the sample, as the yellow areas shown in Fig. 2c, and the total length of the sample except oxides is about $3 \mathrm{~cm}$. After being divided into five parts, five representative positions (the blue areas in Fig. 2c, which is 0.5, 1.0, 1.5, 2.0 and
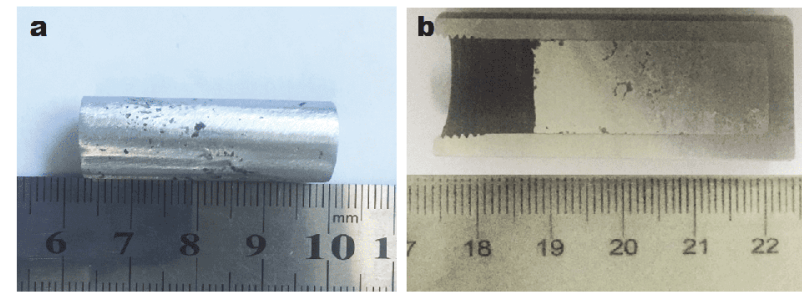

c

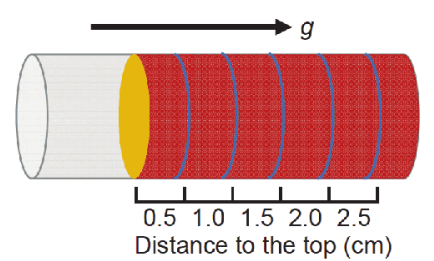

Figure 2 Photographs of Sample 1 before (a) and after (b) supergravity. (c) Schematic of the sampling position (blue areas).

$2.5 \mathrm{~cm}$ from the top, respectively, are chosen, polished, and tested for the following experiments.

Fig. 3 presents the SEM and EDS analysis for the $\mathrm{AlZn}_{0.4} \mathrm{Li}_{0.2} \mathrm{Mg}_{0.2} \mathrm{Cu}_{0.2}$ (Sample 1) entropic alloys. It can be clearly seen from the low-magnification secondary electron images (Fig. 3a) that the alloy is mainly made up of

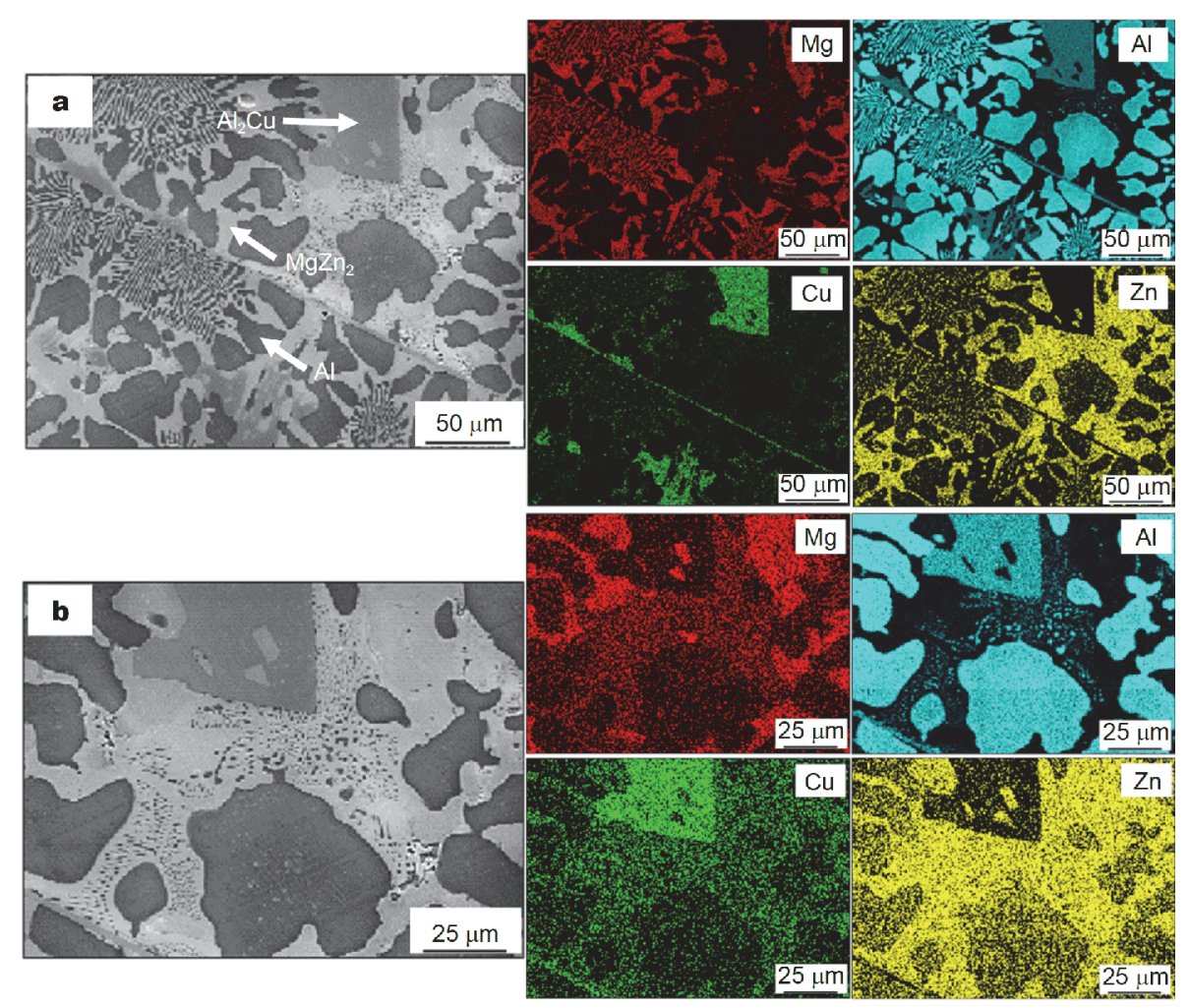

Figure 3 (a) The low-magnification and (b) high-magnification SEM and EDS analysis for the $\mathrm{AlZn}_{0.4} \mathrm{Li}_{0.2} \mathrm{Mg}_{0.2} \mathrm{Cu}_{0.2}$ (Sample 1). 
three phases. Fine binary eutectic microstructures of the $\mathrm{MgZn}_{2} / \mathrm{Al}$ phase (white and black) are formed in the smaller half of the sample and distribute homogeneously, whereas the primarily solidified $\alpha-\mathrm{Al}$ phase (with a dark contrast) can be observed throughout the sample. Moreover, the $\mathrm{Al}_{2} \mathrm{Cu}$ phase displays a block-like shape. The volume fraction of the primary $a-\mathrm{Al}$ phase is estimated as $40 \%$ and the eutectic colony size is approximately $30-50 \mu \mathrm{m}$. Fig. $3 \mathrm{~b}$ presents the highermagnification images showing the existence of the ternary eutectic microstructure of $\mathrm{MgZn}_{2} / \mathrm{Al} / \mathrm{Zn}$. This trend is probably caused by the inhomogeneous distribution of $\mathrm{Zn}$, which precipitates from $\mathrm{Al}$ and contributes to the formation of the ternary eutectic.

Fig. 4 presents the XRD profiles of the three samples, which also reveal the same three-phase microstructure of $\mathrm{Al}, \mathrm{Al}_{2} \mathrm{Cu}$, and $\mathrm{MgZn}_{2}$. It is noted that some diffraction peaks derived from aluminum oxides are also detected in the samples. Moreover, because the composition and the phase formation are complex in the light-weight entropic alloys $\mathrm{AlZn}_{0.4} \mathrm{Li}_{0.2} \mathrm{Mg}_{0.2} \mathrm{Cu}_{0.2}$ [25-27], and the molecular diffusion and mass transfer process increase by hundreds of times in a supergravity field, some unknown ternary or even quaternary phases appear. These unknown phases will be further investigated in our following work.

\section{Graded microstructure and homogeneous composition after supergravity}

The SEM images of graded microstructures for the five positions from all the three samples are presented in Fig. 5. In Sample 1 (Fig. 5a), no centrifugation is applied during insulation and cooling. Therefore, all the three phases distribute uniformly from top to bottom. In Fig. 5b, Sample 2 is centrifuged for 5 min during temperature holding, and the fine binary eutectic $\mathrm{MgZn}_{2} / \mathrm{Al}$ phase is more pronounced, compared with Sample 1. Meanwhile, the primary a-Al's share of all the phases falls down relatively. The volume fraction of the primary $\alpha-\mathrm{Al}$ phase is estimated at ca. (circa) $10 \%$ and the eutectic colony size is larger than $70 \mu \mathrm{m}$. The slightly lower volume fraction of the $\alpha$-Al phase in Sample 2 should be due to the precipitation of the supersaturated solute $\mathrm{Mg}$ and $\mathrm{Zn}$ from the $\alpha-\mathrm{Al}$ phase under the huge gravity. It should be noted that in the bottom part of the sample, the morphology of the eutectic changes from a circle to strip, which is also attributed to the higher centrifugal force, relative to the top. In Sample 3, the supergravity field is applied in the thermal insulation and the following cooling, which means that the effect of both the solidification process and supergravity field needs to be con-

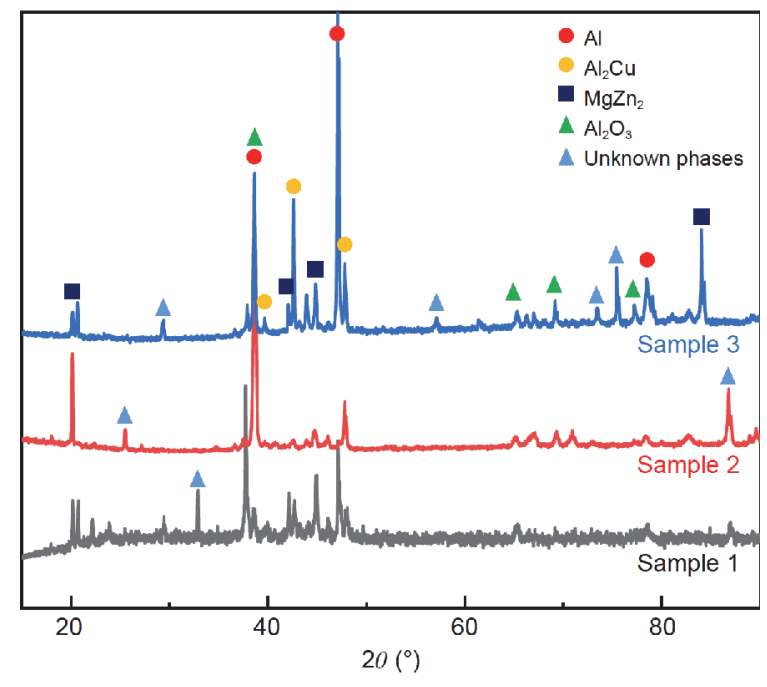

Figure 4 XRD profiles of $\mathrm{AlZn}_{0.4} \mathrm{Li}_{0.2} \mathrm{Mg}_{0.2} \mathrm{Cu}_{0.2}$ entropic alloys.

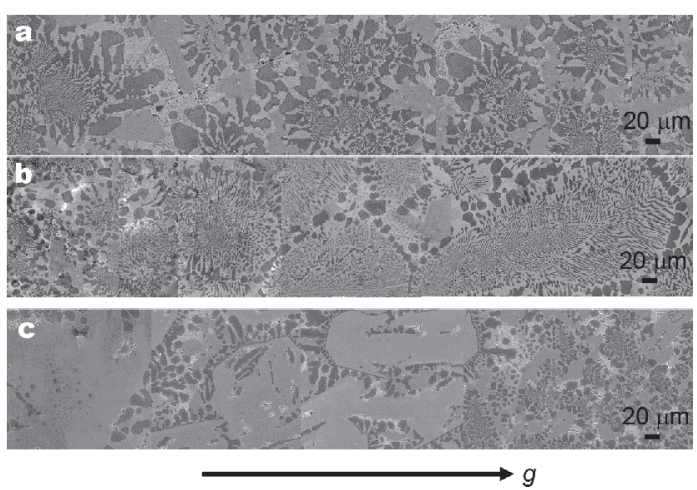

Figure 5 SEM images showing the graded microstructure of (a) Sample 1; (b) Sample 2 and (c) Sample 3.

sidered. This trend causes a more obvious microstructure gradient and promotes the appearance of bulk intermetallics, as can be seen in Fig. 5c. Along the direction of supergravity, the morphology of $\mathrm{MgZn}_{2}$ changes from bulk intermetallics to long-strip shape. Finally, they disappear, and all transfer into eutectic structures. The graded-distributed aluminum oxides, which is due to the supergravity effect, have a significant influence on the solidification of $\mathrm{MgZn}_{2}$, and thus promote the sudden appearance of the bulk $\mathrm{MgZn}_{2}$ phase, compared with the other two samples. Besides, the better flow ability of the eutectic structure, relative to intermetallics, also contributes to the gradient structures.

The contents of $\mathrm{Al}, \mathrm{Mg}, \mathrm{Zn}$, and $\mathrm{Cu}$ in all the five representative positions are determined by the XRF analysis. Fig. $6 \mathrm{a}-\mathrm{c}$ show the elemental distribution of 

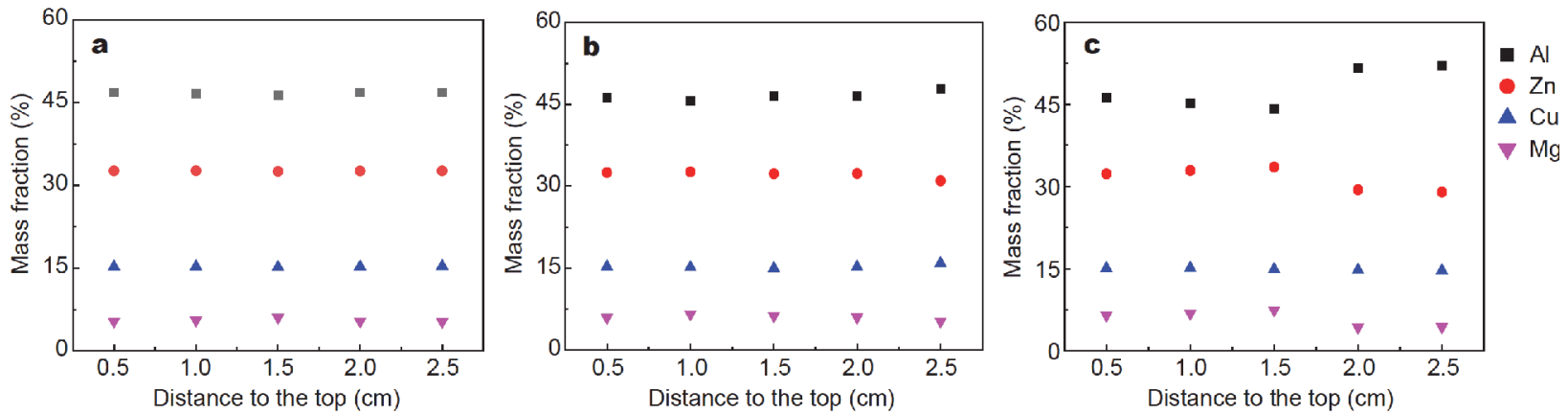

Figure 6 Elemental distributions of (a) Sample 1, (b) Sample 2, and (c) Sample 3.

Samples 1, 2 and 3, respectively. The composition of both Sample 1 and 2 is homogeneous from the top to the bottom, while there are some compositional fluctuations in Sample 3. This trend may be caused by the combined effect of supergravity force, entropic force and the interatomic force, which is explained in the following text.

\section{Hardness of the alloys}

The Vickers hardness values of the selected positions are listed in the Fig. 7. It is noteworthy that all the samples have the hardness values larger than $200 \mathrm{HV}$, and these hardness levels are much higher than those of the conventional $\mathrm{Al}$ cast alloy (AC4D) or $\mathrm{Al}-\mathrm{Mg}-\mathrm{Zn}$ alloy [28]. The hardness value remains unchanged in Sample 1. The hardness of Sample 2 linearly increases from top to bottom with an average hardness of ca. $250 \mathrm{HV}$, reaching its maximum value of $270 \mathrm{HV}$ at the bottom. This gradient hardness values probably results from the increasing centrifugal force, which effectively refines the grains at the bottom. In Sample 3, the hardness value significantly rises relative to Sample 2, because of the formation of

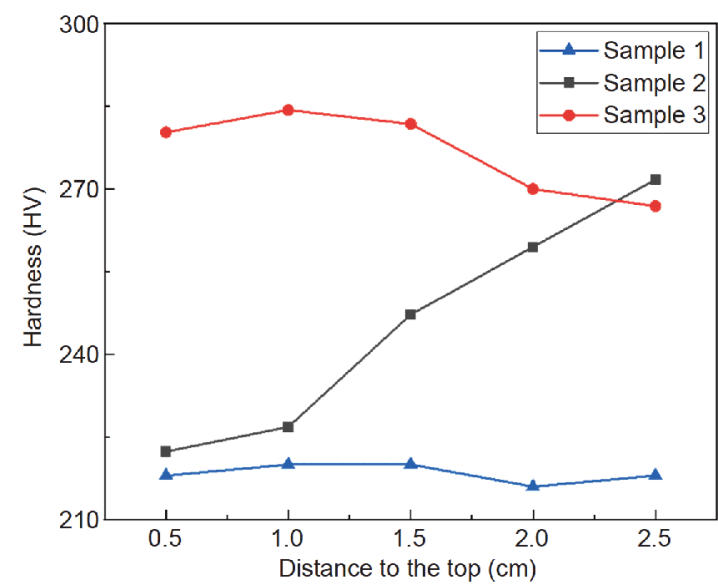

Figure 7 The gradient Vickers hardness value of Samples 1, 2 and 3. high-hardness bulk intermetallic $\mathrm{MgZn}_{2}$ [29], and then it declines in the bottom part resulting from the eutectic structure. There is little difference in the hardness value of the bottom part between Sample 2 and 3, where they share the same eutectic structure. Given the peak of hardness in Sample 3, as larger than $280 \mathrm{HV}$, and the appearance of bulk intermetallics, it can be inferred that Sample 3 has high brittleness and poor plasticity, therefore restricting its scope of application. In term of Sample 2 , the uniformly-gradient hardness values and eutectic structures obviously improve its comprehensive performance.

\section{DISCUSSION}

\section{The effect of supergravity on the composition}

During centrifugation, the redistribution and separation of atoms is more prominent along the centrifugal direction due to the differences between the melting points or densities of the solid particles. Atoms in the system are subjected to the intensive interaction of multiple forces, such as the supergravity, interatomic force, fluid force, entropic force. There are no certain directions of the interatomic force and the entropic force, and the effect of fluid force is ignored as it is too complex.

The entropy force is derived from the effect of entropy. According to the maximum entropy production principle (MEPP), when the entropy of an alloy system is high enough, it tends to stabilize the alloy system [30]. As the total entropy of the alloy system is relatively high compared with the traditional aluminum alloys, the alloy system tends to be stabilized. That is to say, the entropic force tends to obtain the homogeneous composition, and it is believed that its direction depends on the state of the atom in the system.

The quantity differences for the different forces lead to the unique elemental distribution. When the entropic 
force is larger than the supergravity separation force, the homogeneous composition will be obtained, just as Samples 1 and 2. If not, there are some elemental fluctuations, just as Sample 3. The centrifugation is applied during cooling, and the centrifugal time is longer. Hence, the supergravity effect and the elemental density are dominant. The smaller density of $\mathrm{Mg}$, in addition to the strong $\mathrm{Mg}-\mathrm{Zn}$ interatomic force, contribute to element $\mathrm{Mg}$ and $\mathrm{Zn}$ rising to the top, while the heavier elements gathering in the bottom part.

\section{The effect of supergravity on the phase morphology}

There is also a dramatic change of the morphology in Sample 3, compared with the other two samples. Bulk intermetallics appear, and there is an obvious gradient morphology from top to bottom, where the bulk intermetallics are in the top part, while the eutectic structure is in the bottom part. The underlying reasons for the formation of bulk intermetallics are (1) the added element reaching the composition range of the primary crystal compound; (2) the solidification temperature lying in the range of the compound formation; and (3) a sufficient growth time. In the supergravity field, the force applied on the crystals needs to be taken into consideration. During melting and solidification, the enhanced phase transfer and micro-mixing, as well as solute redistribution, result in the inhomogeneous elemental distribution and atomic segregation, which exactly creates conditions for intermetallics' growth. The relatively smaller force applied in the top part of the sample also accelerates their growth. Another reason is the graded oxygen concentration. When the supergravity field is applied during cooling, a small amount of oxide is present in the molten metal as aluminum oxides, and they are crushed by the huge centrifugal force and the strong liquid convection. In the meantime, they rise to the upper part of the sample with the negative direction of supergravity, simply due to the density differences with the molten metal. Finally, they distribute gradiently from top to bottom, which then act as a heterogeneous nucleus for the formation of bulk $\mathrm{MgZn}_{2}$. The schematic of the gradient change in the oxide content is shown in the red line of Fig. 8b. Except for the above analysis, it can also be deduced that the eutectic structure has a better flow ability than intermetallics. As a result, they flow to the bottom of the sample and fill the seam along the direction of supergravity.

In Sample 2, when the supergravity field is applied during temperature holding, the proportion of each phase changes, maybe just because there is no graded oxygen concentration during nucleation and growth.
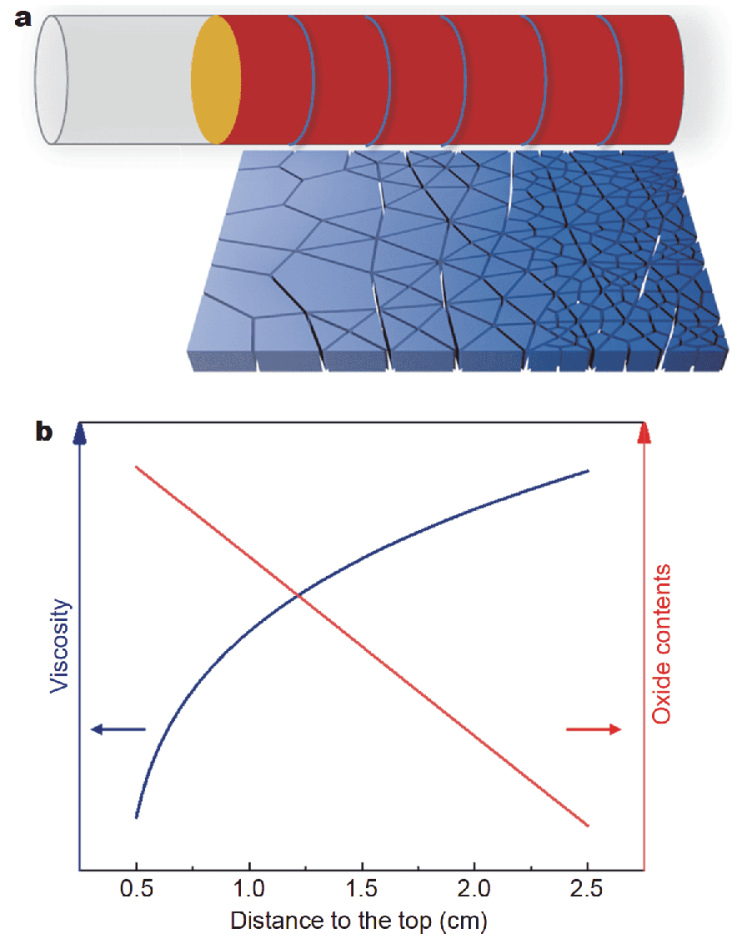

Figure 8 The gradient change in (a) grain size; and (b) viscosity and oxide content under supergravity.

\section{The effect of supergravity on the gradient grain size}

The molten metal is subjected to extreme forces in the supergravity field, which has a huge impact on microstructures and performances [31]. The resultant force $(F)$ applied on the micro-volume unit at $r$ position contains a vertical gravity and a horizontal supergravity force, described as the following function:

$F=\sqrt{(m g)^{2}+\left(m \omega^{2} r\right)^{2}}$,

where $r=0.25-0.28 \mathrm{~m}$ from top to bottom of the sample in this experiment. The resultant force can generate resultant pressure, and therefore accelerate the particles motion. The pressure, $p(\mathrm{~Pa})$, applied on per unit volume can be calculated via:

$$
\begin{aligned}
P & =\frac{F}{S}=\sqrt{\frac{(m g)^{2}+\left(m \omega^{2} r\right)^{2}}{S^{2}}} \\
& =\rho \sqrt{g^{2}+16 \pi^{4} n^{4} r^{2}},
\end{aligned}
$$

where $S$ is the cross-sectional area of the micro-volume unit, $\rho\left(\mathrm{g} \mathrm{cm}^{-3}\right)$ is the density of the molten metal, and $n$ is the rotational speed $\left(\mathrm{r} \mathrm{s}^{-1}\right)$, which has the value of $22.3 \mathrm{r} \mathrm{s}^{-1}$ when the supergravity coefficient of $G=500$. Note that when the samples are centrifuged at an extreme speed, $g^{2}$ is much less than $16 \pi n^{4} r^{2}$. As a result, the net 
pressure applied on per unit volume is proportional to the density of the molten metal, the rotational speed, and the distance to the centrifugal axis, as shown in Equation (4):

$P=4 \rho \pi^{2} n^{2} r$.

Based on the above formula, the resultant pressure gradient applied on the micro-volume unit from top to bottom is calculated as $4.94-5.33 \mathrm{kPa}$ in this experiment. This extremely strong pressure not only has an effect on the grain refinement, but also results in the viscosity change.

Assuming that all the molten metal transfers into the ideal alloy gas, which is at the constant boiling point $\left(T_{\text {vap }}\right)$ and is at the standard atmospheric pressure $\left(P_{0}\right)$, and combining with the laws of thermodynamics:

$\mathrm{d} U=T \mathrm{~d} S-p \mathrm{~d} V$,

and the equation of the ideal gas equation:

$p V=n R T$,

the entropy value of those ideal alloy gas, $S_{0}$, can be calculated as:

$S_{0}=n C_{1} \ln T_{\text {vap }}+n \ln P_{0}$,

where $n$ is the moles of gas (mol), $C_{1}$ is the molar heat capacity of the gas $\left(\mathrm{J} \mathrm{mol}^{-1} \mathrm{~K}^{-1}\right)$, and $R$ is the gas constant as $8.314 \mathrm{~J} \mathrm{~mol}^{-1} \mathrm{~K}^{-1}$. After transferring back into the molten metal at $T_{\mathrm{e}}=680^{\circ} \mathrm{C}$, the entropy value of the system decreases as:

$S=-n R \ln p-A$,

where $A$ is a complex constant, and $A=S_{0}-n C_{2} \ln \frac{T_{\text {vap }}}{T_{\mathrm{e}}}-\frac{\Delta H_{\text {vap }}}{T_{\text {vap }}}$ in which $C_{2}$ is the molar heat capacity of the liquid, and $\Delta H_{\text {vap }}$ is the latent heat of vaporization. The change in entropy results in a change in the viscosity $(\eta)$ of the liquid, according to the AdamGibbs function of $\eta=\eta_{0} \exp \left(\frac{c}{T S_{\mathrm{c}}}\right)$ where $c$ is a constant, and it can be concluded that

$$
\eta=\eta_{0} \exp \left(\frac{c}{T_{\mathrm{e}}(-n R \ln p-A)}\right) \text {. }
$$

With the pressure value calculated as $4.94-5.33 \mathrm{kPa}$ through Equation (4), the gradient variation of the liquid viscosity coefficient can also be obtained, described as the blue line in Fig. 8b. Therefore, as the pressure increases in the melt, the viscosity increases, leading to a decreased diffusion coefficient of $D_{\text {eff }}$, based on the Stokes-Einstein relationship:

$D_{\text {eff }}=\frac{k T}{3 \pi \eta a}$, where $a$ is the interatomic distance. According to the solidification principle, the grain growth in the melt undergoes nucleation and growth. An increase in melt viscosity hinders the atomic diffusion, and the rate at which atoms move toward the nucleus slows down, which causes the grain to decrease.

As a result, because of the linearly increased pressure value and decreased diffusion coefficient, the grain size decreases gradually along the supergravity direction, which in turn increases the hardness in Sample 2. The gradient change in grain size is also shown in Fig. 8a.

\section{CONCLUSIONS}

The Al-Zn-Li-Mg-Cu light-weight entropic alloys with different kinds of graded microstructures have been successfully prepared under the supergravity field. When the supergravity field is applied during temperature holding, the proportion of each phase changes. When alloys are centrifuged during cooling, the morphology of $\mathrm{MgZn}_{2}$ significantly changes from the bulk intermetallics to eutectic structures along the direction of supergravity. There are slight compositional-fluctuations in the alloy, due to the dominant entropic force among the multiple forces. The graded hardness is obtained through supergravity, just because of the gradient variation of pressure and viscosity which refines the grains.

Received 15 August 2018; accepted 9 October 2018;

published online 8 November 2018

1 Koizumi M. FGM activities in Japan. Compos Part B-Eng, 1997, 28: $1-4$

2 Gupta A, Talha M. Recent development in modeling and analysis of functionally graded materials and structures. Prog Aerospace Sci, 2015, 79: 1-14

3 Adachi S, Ueda N. Combined plasma carburizing and nitriding of sprayed AISI 316L steel coating for improved wear resistance. Surf Coatings Tech, 2014, 259: 44-49

4 Suresh S. Graded materials for resistance to contact deformation and damage. Science, 2001, 292: 2447-2451

5 Shanmugavel P, Bhaskar GB, Chandrasekaran M, et al. An overview of fracture analysis in functionally graded materials. Euro J Sci Res, 2012, 68: 412-439

6 Fang TH, Li WL, Tao NR, et al. Revealing extraordinary intrinsic tensile plasticity in gradient nano-grained copper. Science, 2011, 331: 1587-1590

7 Wei Y, Li Y, Zhu L, et al. Evading the strength-ductility trade-off dilemma in steel through gradient hierarchical nanotwins. Nat Commun, 2014, 5: 3580

8 Kawasaki A, Watanabe R. Concept and P/M fabrication of functionally gradient materials. Ceramics Int, 1997, 23: 73-83

9 Zhu J, Lai Z, Yin Z, et al. Fabrication of $\mathrm{ZrO}_{2}-\mathrm{NiCr}$ functionally graded material by powder metallurgy. Mater Chem Phys, 2001, 68: 130-135

10 Zhang $\mathrm{Y}$, Han J, Zhang X, et al. Rapid prototyping and combustion 
synthesis of TiC/Ni functionally gradient materials. Mater Sci EngA, 2001, 299: 218-224

11 Groves JF, Wadley HNG. Functionally graded materials synthesis via low vacuum directed vapor deposition. Compos Part B-Eng, 1997, 28: 57-69

12 Löffler JF, Bossuyt S, Peker A, et al. High-temperature centrifugation: a tool for finding eutectic compositions in multicomponent alloys. Appl Phys Lett, 2002, 81: 4159-4161

13 Löffler JF, Johnson WL. Crystallization of $\mathrm{Mg}-\mathrm{Al}$ and Al-based metallic liquids under ultra-high gravity. Intermetallics, 2002, 10: 1167-1175

14 Löffler JF, Peker A, Bossuyt S, et al. Processing of metallic glassforming liquids under ultra-high gravity. Mater Sci Eng-A, 2004, 375-377: 341-345

15 Meng L, Gao J, Zhong Y, et al. Supergravity separation for recovering $\mathrm{Pb}$ and $\mathrm{Sn}$ from electronic waste. Separation Purification Tech, 2018, 191: 375-383

16 Wang Z, Gao J, Shi A, et al. Recovery of zinc from galvanizing dross by a method of super-gravity separation. J Alloys Compd, 2018, 735: 1997-2006

17 Wang Z, Gao J, Meng L, et al. Recovery of zinc from Zn-Al-Fe melt by super-gravity separation. ISIJ Int, 2018, 58: 1175-1177

18 Zhao L, Guo Z, Wang Z, et al. Removal of low-content impurities from Al by super-gravity. Metall Materi Trans B, 2010, 41: 505-508

19 Xie Y, Liu C, Zhai Y, et al. Centrifugal casting processes of manufacturing in situ functionally gradient composite materials of Al19Si-5Mg alloy. Rare Met, 2009, 28: 405-411

20 Li RX, Liaw PK, Zhang Y. Synthesis of $\mathrm{Al}_{x} \mathrm{CoCrFeNi}$ high-entropy alloys by high-gravity combustion from oxides. Mater Sci Eng-A, 2017, 707: 668-673

21 Kumar A, Gupta M. An insight into evolution of light weight high entropy alloys: a review. Metals, 2016, 6: 199

22 Stepanov ND, Shaysultanov DG, Salishchev GA, et al. Structure and mechanical properties of a light-weight AlNbTiV high entropy alloy. Mater Lett, 2015, 142: 153-155

23 Youssef KM, Zaddach AJ, Niu C, et al. A novel low-density, high- hardness, high-entropy alloy with close-packed single-phase nanocrystalline structures. Mater Res Lett, 2015, 3: 95-99

24 Tseng KK, Yang YC, Juan CC, et al. A light-weight high-entropy alloy $\mathrm{Al}_{20} \mathrm{Be}_{20} \mathrm{Fe}_{10} \mathrm{Si}_{15} \mathrm{Ti}_{35}$. Sci China Technol Sci, 2018, 61: 184-188

25 Yang X, Chen SY, Cotton JD, et al. Phase stability of low-density, multiprincipal component alloys containing aluminum, magnesium, and lithium. JOM, 2014, 66: 2009-2020

$26 \mathrm{Du} \mathrm{XH}$, Wang R, Chen C, et al. Preparation of a light-weight MgCaAlLiCu high-entropy alloy. KEM, 2017, 727: 132-135

27 Li R, Gao JC, Fan K. Study to microstructure and mechanical properties of Mg containing high entropy alloys. MSF, 2010, 650: 265-271

28 Teng GB, Liu CY, Ma ZY, et al. Effects of minor Sc addition on the microstructure and mechanical properties of $7055 \mathrm{Al}$ alloy during aging. Mater Sci Eng-A, 2018, 713: 61-66

29 Takata N, Okano T, Suzuki A, et al. Microstructure of intermetallic-reinforced Al-Based alloy composites fabricated using eutectic reactions in $\mathrm{Al}-\mathrm{Mg}-\mathrm{Zn}$ ternary system. Intermetallics, 2018, 95: 48-58

30 Zhang Y, Zuo TT, Tang Z, et al. Microstructures and properties of high-entropy alloys. Prog Mater Sci, 2014, 61: 1-93

31 Yoshikawa T, Morita K. Refining of Si by the solidification of Si-Al melt with electromagnetic force. ISIJ Int, 2005, 45: 967-971

Acknowledgements Zhang Y would like to thank the financial support from the National Natural Science Foundation of China (NSFC, 51471025 and 51671020).

Author contributions Zhang $\mathrm{Y}$ and Guo $\mathrm{Z}$ designed the experiments; $\mathrm{Li} \mathrm{R}$ and Wang $\mathrm{Z}$ performed the experiments; Zhang $\mathrm{T}$ and Li L contributed to the characterizations; Li R wrote the paper with support from Liaw PK. All authors contributed to the general discussion.

Conflict of interest The authors declare that they have no conflict of interest. 


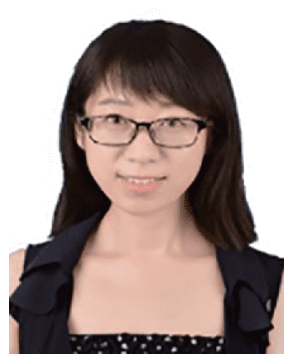

Ruixuan Li is a PhD student at the State Key Laboratory for Advanced Metals and Materials, University of Science \& Technology Beijing (USTB), under Prof. Zhang's supervision. Her interest focuses on the aluminum-based mediumentropy alloys with high strength.

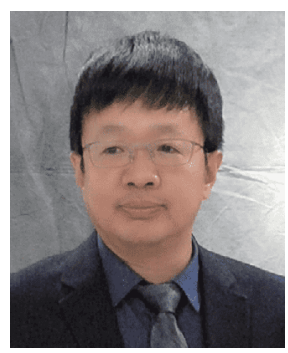

Yong Zhang has been a professor of materials science at the USTB since 2004. He has published over 200 papers, including two review papers, each of which is about 100 pages in the journal of "Progress in Materials Science (PMS)". He proposed a parameter to evaluate the configurational entropy effect over the enthalpy effect above the melting temperature, and it has been verified effective to predict the phase formation for the multicomponent materials.

\title{
超重力场下Al-Li-Mg-Zn-Cu多组元合金的梯度结构
}

\author{
李蒿轩 ${ }^{1,2}$, 王哲 ${ }^{3}$, 郭占成 ${ }^{3}$, Peter K. Liaw ${ }^{4}$, 张涛 ${ }^{5}$, 李扬德 ${ }^{5}$, 张勇 $^{1,2^{*}}$
}

摘要 研究梯度材料的组织结构及其性能可以加速开发具有优异性能的新成分材料. 本文提出一种新方法来制备梯度多组元合金Al-Li$\mathrm{Mg}-\mathrm{Zn}-\mathrm{Cu}$ —超重力法(即离心). 通过实验条件的优化和系统的表征, 我们发现超重力处理后出现了梯度组织结构和硬度值. 沿着超重力 方向, 合金的组织结构从大块金属间化合物转化为共晶结构, 同时铝的氧化物也在离心中被打碎并沿着这一方向梯度分布. 实验结果表示, 通过短时间离心的超重力方法有望提升合金综合性能并加快高性能多组元合金的开发. 